\title{
Di Çarçoveya Bîra Civakî Ya Kurdan De Têkiliya Bûyerên Dîroka Nêzîk û Kilamên Dengbêjan
}

\author{
In the framework of the memory of the Kurdish community Dengbejs's songs about the recent \\ events
}

Tekin Çiftçi ${ }^{1}$

Received: November 06, 2015

Accepted: December 12, 2015

\begin{abstract}
Kurte
Çanda devkî aliyekî bîra civakan e. Bi taybetî jî di civakên ku nivîs bi têra xwe pêş neketiye de vegûhestina çandî piranî bi rêbaza devkî pêk tê. Ev pevbestiya devkî bi demê re diguhere wêjeya devkî û muzîkê. Di çanda kurdan de jî barkêşên sereke yên vê çandê dengbêj in. Bikarneanîna zimanê kurdî ya bi awayekî fermî ji bo pêşketina dîroka nivîskî ya kurdan bûye astengekê, lê li aliyê din dengbêjên kurdan ji bo ku ev bûyer û qewimînên dîrokî ji bîra cîvakê neçin, her yej jin wan kirine mijara kilaman û vî rengî bîreke civakî ava kirine. Li her deverê Kurdistanê kilamên bi vî rengî hene. Her yek ji wan mijara lêkolîneke serbixwe ye. Dengbêj bi vê peywira xwe veguhestina dîrok û bîra kurdî ya bi rêya muzîkê pêk tînin. Bi taybetî ji kilamên şînê gencîneyeke qirase ya muzîkê saz bûye. Dengbêj bi strandina van kilaman bûne barkêş û berdevkên dîroka nêzîk ya civaka kurdî. Di vê xebatê te du kilamên kurdî tevî çîrokên wan û dîroka wan wekî mînak hatine dayîn. Armanca vê xebatê ew e ku wate û girîngiya muzîka kurdî ya dengbêjiyê ya di bîra çandî ya kurdan de ji aliyê dîrokê ve bê nîqaşkirin. Di beşa dawî de jî bi kurt û kurmancî li xebatên pişaftina muzîka kurdî hatiye sekinîn.
\end{abstract}

Miftepeyv: Çanda Devkî, Dengbêj, Bûyer, Kilam, Dîrok

\begin{abstract}
Oral culture constitutes an aspect of social memory. Especially in the societies where written culture has not developed enough, cultural transmission comes true in an oral way. This oral attitude transforms into music and oral literature over the time. In the Kurdish music, Dengbejs are the primary transmitters of this oral culture. As Kurdish has not been used as a formal language for a long time, it has prevented Kurdish history to be transferred in written form. On the other hand Kurdish dengbejs, in order not to let historical events to be erased from the social memory, they've made these events up by making each of them a subject of their kllams and in that way built the social memory. In every corner of Kurdistan it's possible to come across to this kind of songs. Each of these can be a subject to a research individually. Dengbejs, with the songs they made, have taken the role to transmit the social consciousness. Particularly from the mournings there have emerged a giant archive. This study aims to make a debate on the importance of music in the Kurdish oral culture in terms of history. In the study, music and history relationship will be discussed in the lights of two different songs which express two different events happened in our recent history. And in the last section, the efforts to assimilate Dersim's history and music will be addressed briefly.
\end{abstract}

Keywords: Oral Culture, Dengbêj, Event, Song, History, Assimilation

Recommended citation:

Ciftci, T. (2016). Di çarçoveya bîra cıvakî ya kurdan de têkılıya bûyerên dîroka nêzîk û kılamên dengbêjan [In the framework of the memory of the Kurdish community Dengbejs' songs about the recent events]. International Journal of Kurdish Studies 2 (1), pp.1 - 10.

\footnotetext{
${ }^{1}$ Corresponding Author: Zanîngeha Mardin Artukluyê Enstitûya Zanistên Civakî Makezanista Dîrokê Bernameya Doktorayê (Mardin Artuklu Üniversitesi Sosyal Bilimler Enstitüsü Tarih Anabilim Dalı Doktora Programı) E-mail: tekincifci02@gmail.com
} 


\section{FOLKLOR Û DÎROK}

Zanîna derbarê hemû çalakiyên mirovan de tenê bi şopandina bermayiyên wan çêdibe. Ev bermayî çi kevirek be, çi peyveke ku çandê nîşan dide be, ferq nake. Lewma jî hêza mezin, pergala serdest, dema ku bixwaze civakekê ji hişmendiya netewî û çandî bêpar bihêle, serî li rêbaza danjibîrkirina bi pergal û dûvdirêj dide (Connerton, 1998). Ango karê xwe bi zanetî û planên demdirêj dimeşîne. Wisa dike ku gelek mirov bi pêvajoyê jî nahisin. Lê ew plan her carê bi ser nakeve. Vegotina serpêhatiyan, du tiştên girîng bi hev re çê dike. Yek jê, liv û tevgera kesên ku qala wan dibe, ya din jî bi vegotina serpêhatiyê re eşkerekirina rewş û dema bûyerê ye (Connerton, 1998) Li gor Halbwachs, kes serpêhatiyên xwe bi riya endamtiya civakê, merivantî, bawerî û têkiliyên çînî bi bîr tînin. Dema ku em serî li bîra civakê didin, rewş û dema bûyerê bi civaka wê demê re tên bîra me.

Yek ji navgînên domandina folklorê, bîra civakî û rê û rism in. Du cureyên rê û risman yên sereke hene. Yek jê rê û rismên olî ne, ya din jî rê û rismên girêdayî salê, salvegerê û jiyana rojane ne. Rê û rismên olî, ji ber ku nivîskî ne, bi hezar salan dikarin bê guhertin bidomin. Her rê û rismek di nava xwe de bîreke civakî û çalakiyeke kevnare dihewîne. Dîrok bi vî rengî dijî. Pîrozbahiyên cejnê, rojî û rê û rismên veşartina miriyan tenê çend mînak in. Rê û rismên girêdayî jiyana rojane, li gor civakan, herêman, demê û pesşketinên teknolojîk diguherin. Cil û bergên folklorî û dawet mînakên van in. Heke em dawetên berê û yên niha bidin ber hev em ê gelek guherînan bibînin.

Dîroka kurdan jî piranî di nava folklorê de dijî. "Min dîroka kurdan ji kilaman hîn kiriye. Kilamên kurdî ji belegeyên fermî saxlemtir in." dibêje lêkolîner/nivîskar Ahmed Aras (Çîfçî, 2012). Kesên ku di kilaman de navê wan derbas dibin ne kesên razber in. Ji vegotina bûyeran tê fêmkirin ku ew, kesên dîrokî ne, bi goşt û hestî ne. Neviyên gelekan hê jî li ser darê dinyayê ne. Digel ku di kilaman de bûyer bi rêzê nayên vegotin jî̀, em bersiva pirsên wekî, şer li ku derê qewimiye, di navbera kîjan aliyan de qewimiye, encam çi bûye û di şer de kî miriye $\hat{u}$ kî maye? dibînin.

Di nav kurdan de, di warê kilamên şeran de, hosteyê herî mezin yê sedsala bîstan, bêguman Karapetê Xaço (1902-2005) ye (Kevirbirî, 2009a). Wek mînak, Karapetê Xaço yek ji wan dengbêja ku bûye şahidê şerê nav eşîrên kurdan û bi kilamên xwe ew dîroka kurda ya nenivîsandi li ser pelên çanda kurdî bi tîpên zêrîn nivîsandiye. Ew şerê ku li herêma Xerzan di navbera eşîrên Reşkotan û Etmankîyan de pêk hatiye bi xêra kilamên wî bi nav û deng bûye. Bi dengê Karapetê Xaço gihaye heta roja îro û dê her bimîne (Karacan ve Nayman, 2014). Karapetê Xaço -ku bi eslê xwe ermenî ye- li herêma Xerzan ji dayik bûye û demekê li mala Filîtê Quto dengbêjî jî kiriye. Xaço, bi kilamên weke Filîtê Quto û Usivê Seydo (Kevirbirî, 2009b), derbarê rewşa herêma Xerzan ya serê sedsala bîstan de gelek agahiyan dide me.

Di folklorê de hêza kevneşopiyê gelekî zêde ye. Hunera devkî, ji ber ku bi awayekî nivîskî nikare bê qeydkirin, heta gelek salan bi riya folklorê dikare bê parastin. Gelek caran ji bo bîranînê, serî li alikariya folklorê tê dayîn (Sokolov, 2009). Li gor lêkolîner/nivîskar Ahmed Aras, bûyerên ku di kilamên dengbêjan de hatine vegotin, ji sedî sed rast in. Mînaka vê yekê ya herî nêzik jî şerê mala Ûsivê Seydo ye. Aras dibêje ku: 
Piştî ku min li kilamê guhdarî kir, ez bi pey belgeyên fermî yên derbarê vê bûyerê ketim. Min hin belge bi dest xistin. Min dît ku kilam û belge wekî hev in. Di ser de, hurgiliyên kilamê zêdetir in. Heke hûn ji min bawer nakin, neviyên wan jî hîn sax in, herin jê bipirsin. Ew dikarin gotinên min piştrast bikin.(Çifçi, 2014).

Di têkiliya folklor û dîrokê de, ji bilî kilaman, berhemên devkî yên wekî çîrok û destanan jî çavkaniyên baş in. Lê belê li vir me xwest ku, di çarçoveya kilaman de wekî destpêkekê, çend agahiyan bidin.

"Folklor hêzeke ku civata bê xwendin $\hat{u}$ nivîsandin bi hev ve girê dide ye." dibêje Mehmed Uzun (Uzun, 2006). Rûmeta berhemên folklorî, bi jêhatîbûna vebêj ve girêdayî ye. Kî dikare bibêje ku berhemên nivîskî bêalî ne? Berhemên devkî ne bêalî ne; çêkirî ne, guherbar in $\hat{u}$ alîgir in. Yek ji aliyê dîroka devkî ya baş, derfeta bihevredîtina rastiya şexsî $\hat{u}$ civakî ye. Ji serhatiyan, derfeta dîtina sazkirina pira navbera bîra şexsî ̂̀ ya civakî çê dibe (Çağlayan, 2010).

Berhemên devkî ji bo dîroka gelên ku hê nebûne dewlet, çavkaniyên ferz in. Her ku berhem derbasî ser kaxizê bibin ê ev taybetmendî jî ji holê rabe. Lê heya wê demê divê ev berhem bêne parastin. Li aliyê din ve tu nivîs nikare cihê "deng" bigire. Lewma jî rêya parastina berhemên devkî ya herî baş, qeydkirina "deng"e (Porteli, 2005, r.222). Lê mixabin temenê teng berê bi temenê mirovan ve girêdayî bû. Ji ber kêmasiya derfetên teknolojîk îro em nikarin bigihêjin dengê dengbêjên navdar. Ew yek ji bo gelê kurd jî wisa ye. Gelek dengbêj behsa xweşbûna dengê dengbêjê navdar Evdalê Zeynikê dikin. Gelek dengbêj kilamên wî dibêjin û hinek kesan jî kilamên wî ji devê dengbêjên din girtine û nivîsandine. Lê mixabin, em çiqas berhemên Evdalê Zeynikê bixwînin jî, em ê tu caran bi tahma dengê Evdal nehisin.

Rastbûna bûyereke devkî li gor bîr, zanîn û ceribandina şexsî diguhere. Lewma jî carinan rastî û fantezî tevli hev dibin. Lê her çi be jî divê ew serhatî, wekî dewlemendiyeke çandî û bîra civakî bêne dîtin û parastin. Çanda kurdî, him bi cihêrengiya xwe, him jî bi teşe û naveroka xwe gelekî dewlemend e. Li dijî astengiyên siyasî, çandî, derûnî û aborî, mayîna ziman û çanda kurdî ya heta îro, bi tevkariya helbestvanên wekî Ehmedê Xanî ku berhemên nivîskî dane û bi dengbêjên wekî Evdalê Zeynikê bûye. Di berdewama jiyîna wêjeya kurdî de divê mirov keda ku malbata Bedirxaniyan li mişextiyê dane jî ji bir neke (Erbay, 2009).

Bûyerên trawmatîk (wekî Terteleya Dêrsimê), di nav komekê de hestên hevpar çêdikin û ew trawma di nav komê de nasnameyeke hevpar ava dike. Ew formasyon bandorê li ser nasnameya şehsî jî dike (Çelik, 2015). Mirov bi zimanê xwe, hisên xwe, xeyalên xwe û hêrsên (xeyd) xwe bi lêv dike (Ceweri, 2004). Di belavbûn û xurtbûna wêjeyê de rola desthilatiyê jî gelekî zêde ye. Desthilatî li ku belav bûbe, wêje jî li wê derê pêş ve çûye. Ji ber ku li herêma Kurdistanê desthilatiya kurdan qels maye wêjeya kurdî ya nivîskî jî qels maye. Wek mînak, berhemên kurdî ku heta îro ji bo zimanên din hatine wergerandin gelekî kêm in. Çi berhemeke kurdî xelateke Nobelê ne stendiye. Li aliyê din ev nayê wê wateyê ku çanda kurdî ya devkî jî qels e. 
Kilamên kurdî ku heta îro bi awayekî devkî jiyane diyar dikin ku kurd -di sînorên teng de be jî- bune xwediyê desthilatiyê. Dema ku mirov li dengbêjan guhdarî dike û li çîrokên wan kilaman dinihêre, dibîne ku di wir de qala mîr û began tê kirin. Kilamên şînê, ji xeynî êşa ku hatiye kişandin gelek caran di derbarê navên kesan, buyeran, herêman û xwezayê de jî agahiyên hurgiliyan didin me. Yek ji peywira muzîkê ew e ku, dilê mirov rehet dike. Bêhna mirov dertîne û hestên cûda di dilê mirov de peyde dike. Kilam dibin berdevkên hestên mirovan yên xweş û ne xweş. Ji vî aliyî ve kilamên şînê, di heman demê de dermanê birînan e, birînên kur dikewînin; êşên ku nayên şîrovekirin vedibêjin (Elaldı, Bilgin, 2011). Kilam dibin rêberê rewşenbîr û nivîskaran. Hin nivîskar û lêkolînerên kurd jî, ji van kilam, destan û çîrokan berhemên wêjeyî yên nivîskî afirandine. Romana Dewrêş̧ê Evdî ya Eyüp Kıran (Kiran, 2011) û berhema lêkolînî ya Ömer Uluçay a bi navê Derwêşê Evdî Destanı (Uluçay, 2006), çavkaniya xwe ji destana Derwêş̧ê Evdî, ku heta îro ji aliyê gelek dengbêjan ve hatiye vegotin, girtine.

Kilamên Kurdî jî -bi taybetî jî kilamên şînê û şeran- wekî kilamên gelên din, bûyerên ku di dîrokê de hatine jiyîn bi awayekî hunerî radigihînin roja me. Helbet bi hezaran kilamên ku li ser şer û şînên ku pêkhatine hatine afirandin hene lê derfetên vê mijarê rê nadin ku em van hemuyan binirxînin. Lewma jî emê li jêr li ser du mînakan bisekinin. Bi vî rengî em ê têkiliya buyerên dîroka nêzik ya kurdan û kilamên ku dengbêjan li ser van bûyeran afirandine derxin holê. Ji van yek li bi zaravayê kurmancî ye. Ya din jî bi zaravayê zazakî/kirmanckî ye.

\section{Kilama Nîdayî Beg (Nemînim) û Dîrok}

Divê mirov bi kûrahî li ser dengbêjiyê bisekine. Lewre dengbêjî dîroka gel ya nêzîk radixe ber çavan. Her kilameke dengbêjan bi taybetî jî kilamên şînê, beşek ji dîroka devkî ye. Dîroka devkî ji aliyekî ve ji bo fêmkirina dîrokê alîkariya me dike; ji aliyê din ve jî ji bo dahatûyê bîra civakê saz dike (Ersoy, 2009). Assmann dibêje ku bîra civakî, ji xeynî sazkirina rabirdûyê xwediyê hêza bi hev ve girêdana dema niha û dema bê ye jî (Assman, 2001). Dema ku mirov têkeve nava kûrahiya dengbêjiyê mirov dê gelek tiştan bibîne. Yek ji van şahidbûna kilaman ya dîrokê ye. Ji ber ku zimanê nivîskî di nava civata kurdan da zêde belav nebûye, dîroka şerê nava dagirker û eşîran, yan jî şerê eşîrên li dijî hev û sedemên wan tevahî bi devkî ango bi çîrok û kilaman gihişstiye roja me. Lewma jî lêgerîn û lêkolînên ku li ser kilamên kurdî çêbibin, ji bo nasîna kevneşopî, wêje, huner, erdnîgarî, jiyana manewî û dîroka kurdan ê bibin çavkaniyên girîng (Veroj, 1997). Hebûna nav û cihên ku di nava kilaman de derbas dibin, derbarê dem û cihê serpêhatiyan de agahiyan dide me (Oğuz, 2010). Dibe ku hemî ne rast bin û zêdekirin tê de hebin. Ew tiştekî asayî ye. Lewre, kilam jî bi demê re diguherin. Lê ev yek nikare rastiya hebûna bûyerê ji holê rake. Her ku dema qewimîne kevin dibe û mirov ji herêma qewimînê bi dûr dikeve di kilamê de jî guhertin çêdibin lê dîsa jî hin agahî wek rastî hebûna xwe diparêzin. Gelek dengbêjên kurd, li ser şer û qewimînên ku di navbera dewleta Tirkiyeyê û eşîrên kurdan de pêk hatine, kilam afirandine. Ji ber ku gelek bûyeran di arşîva dewletê de cih negirtine û arşîvên heyî jî ji gel re nehatine vekirin, çavkaniyên kurdan yên herî zindî kilam in. Kilama Reso ya bi navê "Nemînim" ji bo peyitandina vê angaşta me mînakeke baş e. Em dikarin bi alîkariya vê kilamê bûyereke dîrokî ya dema nêzik ronî bikin. 
Xwedê belkî kula bixe mala Nîdayî Begê,

Nîdayî Beg ji êvar da sekiniye derê hepsa Bazîdê

Vediqetîne egîd $\hat{u}$ cindiya

Hewşa Şewêş Efendî da

Cinazê egîd $\hat{u}$ xweşmêra ji êvara xwedê $d a$

Xemilandiye di xûnê da bi singûya

Herçî kuştin, herçî mayî girtin sirgûn kirin

Berê wan dan Anadolê, Sînopê, Zongildaxê

Dayê nemayê nav girtiya...(Dît, 2010).

Dema ku em hinekî li van nav, herêm û bûyeran dinihêrin, gelek rastî derdikevin pêşiya me.

Nîdayî Beg: Serbaz Nidayi Çakıroğulları,

Bazîd: Navçeya Agirî ye (Doğubeyazıt),

Hepsa Bazîdê: Girtîgeha wê demê,

Hewşa Şewêş Efendî: Şewêş Efendî, kalikê wezîrê perwerdehiyê yê Partiya Demokrat, Sînop û Zongildax: Ji gelek warên ku di dema 1926-1932an de kurd dihatin mişext kirin, du bajarên Tirkiyeyê ne

Wekî ku tê dîtin ji bilî dîroka bûyerê, gelek agahiyên rasterast di kilamê de cih girtine. Ev yek jî diyar dike ku heke lêkolînên baş li ser kilaman werin kirin, wê di derbarê dîroka kurdan ya nenivîsandî de gelek agahî bêne berhevkirin. Bi taybetî jî kilamên şeran her yek bi serê xwe rûpelek ji dîroka kurdan e. Ji aliyê din de, bi teşeya xwe, bi naveroka xwe, bi tevn û hunandina ristan û bi meqam û awaza xwe kilama li jor, ji serî heta binî huner û wêje ye. Hostatiya dengbêj li ber çavan ne. Dibe ku dengbêj qewimîna bûyerê, ji devê hin şahidan bihîstibe. An jî li hin dengbêjên din guhdarî kiribe. Lê belê ev kilam huner bixwe ye û neynika rastiyê ye.

\section{Kilama Findiq Axa û Dîrok}

Digel ku pergala Komara Tirkîyeyê, kurd û çanda wan tune hesibandine jî bi sedan kilamên şînê ku bi alîkariya dengbêjan gihaştine roja îro hene. Kilama Findiq Axayê kurê Qemer Axa yek ji van e. Ev kilam (Hewa'y Findiq Axa) ji aliyê dengbêjê navdar yê Dêrsimê, Silo Qiz ve hatiye gotin. Kilam bixwe bi zaravayê kirmanckî/kirdkî hatiye gotin. Li jêr beşek ji vê kilamê hatiye dayîn. Piştî ku em guh bidin kilamê, em ê bi çend gotinan li ser çîrok û dîroka wê bisekinin.

De bîye bîye’Ăga'e mi bîye

Wuşene Seyd vano: "Teşmîl meve Qemer Ă̆a 
Reyna giredame duzmune sere mîye

Qemer Ăga gosro mine tıfong berze

Reyna giredame duzmune sere mîye"

Wuşene Seyd vano: "Ez zon ke leme

Kafir ma beno binê daraxaciye

Ewro mara pers keno soxurê Kirmanciye*

Zalim mara pers keno soxure Kirmanciye”

Findiq Axa'e mi leme berdo bine daraxacîye

Findiq darê meerzê, sarr bêro şerê cwamerdiye

\section{Hêfê mi bêro cênciya Findiq' $\hat{\imath}$}

Hîre rể la vişîyo têpa erzenê dare

Na dîna kêşte nîna buko dînaka bêtîvare...(Özcan, 2002)

Findiq Axa, lawê yek ji pêşengên eşîra Yusufan, Qemer Axa ye. Qemer Axa di sala 1917-1918an de li dijî Rûsan şer kiriye. Ji ber vî şerî ji aliyê Mustafa Kemal ve hatiye xelatkirin (Bayrak, 1993) Di sala 1936an de Qemer Axa bi hêviya efû kirina dewleta tirk xwe dide dest leşkeran. Ji lawê xwe Findiq Axa jî vê yekê dixwaze. Finfiq Axa berê vê pêşniyarê napejirîne lê piştre ew jî xwe radestî dewletê dike. Lê mixabin rêvebiriya devleta tirk ya wê demê herduyan jî digre û davêje girtîgehê. Bavê wî di sala 1937an de di girtîgehê de dimire. Findiq Axa jî tê dardakirin. Dadgerê wê demê Îhsan Sabrî Çağlayangil jî vê buyerê piştrast dike. Ev kilam jî li ser dîyaloga Wuşenê Seyd û Qemer Axa ku li ser Findiq Axa dikin, hatiye afirandin. Bi kurt û kurmancî, kilam weha dibêje:

"H(w)usenê Seyd dibêje ku: "Were teslîm nebe,

Emê dîsa rextên xwe girêbidin,

Were guh bide min; em ê bi hev re şer bikin.

$H(W)$ usenê Seyd dibêje: "Ez dizanim ew ê me bibin sêdarê

Ew îro pirsa doza kirmanciyê li me dikin.

Findiq Axayê min birine sêdarê

Findiq Axayê darve nekin, bila xelk bê temaşa mêraniyê

Heyfa min tê li xortaniya Findiq Axa

Sê caran benê stûyê wî qetiyaye, lê dîsa darve dikin

\footnotetext{
*Ev peyv, di vê kilamê de bi wateya "pirsgirêka kurd” hatiye bikaranîn [explanation by Tekin Çetin].
} 
Ew dinya ji kesî ra namîne, dinyayeyeke derewîn e.." *

Wekî ku ji kilamê jî tê fêmkirin, Qemer Axa û kurê wî Findiq Axa ji aliyê rêvebiriya wê demê ve bi derewan hatine xapandin û bi fenan hatine girtin. Girtinê jî têrê nekiriye, yek di girtîgehê de ji ber ku nehatiye dermankirin miriye û yê din jî hatiye daliqandin. Sedem çi dibe bila bibe divê mirov neyên daliqandin. Dengbêj ji ber vê rewşê bi hêrs e û xemgîn e. Hêvî dike ku ew kesên ku ev xerabî bi Findiq Axa kirine ê li dinyayê payîdar nemînin û ê rojekê hesabê vî kiryarê xwe yê xirab li hember Xwedê bidin. Wekî ku em ê li jêr jî li ser bisekinin, ew kiryarên hanê ne tesadufî̀ ne. Encamên polîtîkîkayeke çewt in. Siyaseta ku demeke dirêj bi awayekî fermî hatiye meşandin, li ser qirqirina çanda kurdî û zimanê ye. Yek ji qadên pişaftinê ku hatiye diyarkirin jî herêma Dêrsimê ye.

\section{Hewldanên Pişaftina Çand û Dîroka Dêrsimê ya Nêzik}

Di wêjeya kurdî de gelek mînakên kilamên şînê-tevkûjiyê hene. Helbet di nav wêjeya welatên din de jî mirov dikare van mînakan bibîne. Lê belê, ji ber ku kuştin, tevkujî û mişextî wekî çarenûseke reş li pêşiya kurdan hatiye raxistin, di nav wêjeya kurdî ya devkî de mînakên herî bişewat cih girtine.

Li tirkiyê di sala 1936an heta 1952yan ji aliyê saziya Konservatuara Devletê ya Ankarayê ve, bi destê Muzaffer Sarisözen, Halil Bedi Yönetken, li gelek herêman xebatên berhevkirina kilaman hatine meşandin.(Özbek, 1981) Bi taybetî piştî salên 1930î, bi zanetî polîtîkayên asîmîlasyona ziman û çanda kurdan hatiye destpêkirin. Ji bo vê yekê jî xebata pêşîn berhevkirina kilamên kurdî ye. Bi rêya saziyên wekî Halkevlerî (Malên Gel) hin kes hatine herêma Kurdistanê, hunermendên wê demê bi darê zorê dane hev û kilamên wan tomar kirine. Piştre li zimanê wan, nav û naveroka wan kilaman guherandine û kirine tirkî. Di nav van kesan de Ferruh Arsunar kesekî girîng e. Arsunar kilam û beytên Elewî-Bektaşiyê yên kurmancî-kurmanckî dide hev lê bizanetî zimanê wan diqelibîne ser tirkî. Armanc ew e ku çanda kurdî û zimanê kurdî bê înkarkirin. Ji bo ku derewên xwe bidin bawerkirin, bi sedan pirtûk û kovar amade kirine. Di vê mijarê de Yönetken weha dibêje:

"Çiyayên asê, avên bi coş, beyarên xweşik yên Tuncelî, çiqas xwezaya Anadolê bin, çanda muzîkê ya gelên ku li ser vê axê dijîn jî ewqas çanda muzîka tirk ya Anadolê ye. Lewma yî newayên muzîka vê herêmê jî dişibe newaya muzîka herêmên din" (Özcan, 2003).

Lê em baş dizanin ku rastiya çîrokê ne weha ye. Lewre digel hin aliyên hevbeş yên çanda Dêrsimê û çanda tirk hene jî, him ji aliyê bawerî, kevneşopiyan ve him jî ji aliyê ziman ve çandeke xweser ya Dêrsimê heye. Ew gotinên li jor jî diyar dikin ku pergala Cumhuriyetê bi her awayî li ser înkara kurdan hatiye avakirin. Heger armanca wan parastina çanda kurdî ba, divê zimanê van kilaman jî nehata guhertin (Özcan, 2003). Ew rêbaza pişaftinê ne tenê ji aliyê peywirdarên devletê ve hatiye meşandin. Mixabin gelek hunermendên kurd yên wekî

\footnotetext{
* Bi qasî ku min ji kilamê fêm kir, min şîrove kir. Lewre kirmanckiya min pir ne baş e [explanation by Tekin Çetin].
} 
Celal Gûzelses, Îzzet Altinmeşe, Îbrahîm Tatlises jî peyvên kilamên kurdî guherandin û bi vî rengî ew kirin malê çanda muzîka tirkî û bûn şîrikê vî gunehî. Di warê akademîk de jî gelek "rewşenbîrên" kurd û tirk bi berhemên xwe piştgirî dane van xebatên pişaftinê. Wekî mînak Prof. Dr. Kemal Karpat ku çepgir û Kemalîst wî didin ser serê xwe, di berhema xwe ya bi navê "Türk Edebiyatında Sosyal Konular" (Di Edebiyata Tirkî de Mijarên Civakî) de dibêje $\mathrm{ku}$ :

Li hember vê rewşa ku mamosteyekî li Rojhilatê Anadolê vedibêje, helbestvan helbet nikarin xwe ragirin: (Li Bîngolê navê şahiyê tuneye. Min qet kilam nebihîstin. Hevalê min Hanefì Beg sê salin li wir bûye, wî jî kilamek bi tenê jî nebihîstiye (Karpat, 1971).

Mehmet Bayrak di vê mijarê de dibêje ku ew gotinên Karpat, ne rast in. Lewre piştî Karpat bi 10-15 salan ez çûm wê herêmê û min bi tena serê xwe, li herêma Bîngolê ji du sed hebî zêdetir kilamên kirmanckî û kurmancî berhev kirin (Bayrak, 2012). Herweha îro jî bi dehan dengbêjên vê herêmê hene û kilamên xwe dîsa kurdî dibêjin. Digel hemû zor û zextan jî kilamên kurdî li ser lingan e. Mixabin em dikarin bibêjin ku ew polîtîkayên pişaftinê di warê kilam û beytên Elewîtiyê de hinekî bi ser ketine. Bi hezaran beyt, dia û kilamên kurdî (Bi taybetî yên bi zaravayê kirmanckî) wenda bûn; çanda kirmanckî qels bû û di binê malan de riziya. Nifşên nû ji kirmanckî dûr ketin (Elaldı, Bilgin, 2011). Heger îro di ayînên Cemê de zimanê tirkî tê bikaranîn, ew nîşana serkeftina xebatên pergala Cumhuriyetê ye. Divê ev mijar bi berfirehî bê niqaşkirin. Lê sînorên vê xebatê rê nadin vê niqaşê. Helbet xebatên pişaftinê ne tenê bi rêya berhevkirin û guherandina kilaman hatiye kirin. Wek mînak, di sala 1925an de "Şark Islahat Planı" (Plana Sererastkirina Rojhilat) hatiye amadekirin. Piştî vê planê bi sed hezaran kurd ji cih û warên xwe bûne û hatine mişextkirin. Li dibistan û dadgehan axaftina bi kurdî hatiye qedexekirin, perwerdehiya bi kurdî hatiye qedexekirin. Din av pirtûkan de peyva "Kurdistan" hatiye derxistin û hwd.(Bayrak, 2013). Hezar mixabin ku ew polîtîkayên pişaftinê, îro jî (kêm be jî) di qadên cûda de têne meşandin. Ew jî mijara xebateke din e.

Heger em vegerin ser mijara pişaftina çanda kurdî ya bi rêya muzîkê, em dikarin bibêjin ku li dijî xebatên pişaftinê, di salên 1970-1980an de bi pêşengiya dildarên folklora kurdî yên wekî Mesut Özcan û Mehmet Bayrak ev kilamên Dêrsimê tomar kiriye. Bayrak, ev kilamên ku berhev kirine wekî sê bergên qirase, bi navê Kürt Müziği, Dansları ve Şarkıları (Muzîk, Dans $\hat{u}$ Şarqiyên Kurdî), di sala 2002an de çap kiriye (Bayrak, 2002). Di berga pêşî de gotarên gelek kesên hêja, ku di mijara muzîka kurdî de pispor in, cih girtiye. Her du bergên din jî ji textên kilaman û notayên hinekan pêk tên. Mesut Özcan jî bi navê Öyküleriyle Dersim Ağıtları (Özcan, 2002) pirtûkeke ku ji du bergan pêk tê amade kiriye. Îro hejmara xebatên bi vî rengî gihaye hezaran. Ji xeynî van xebatan bi destxistina hin derfetên perwerdehî û teknolojiyê muzîk û kilamên kurdî belav dibin.

\section{WEKÎI ENCAM}

Kilamên dengbêjan yên şînê, bîra civakê saz dikin. Helbet ev sazkirin bi rêyeke estetîk pêk tê. Dengbêj bi kilamên xwe dibin berdevkê êş û azarên ku di demên berê de hatine jiyîn. Serpêhatiyên şexsan dikin malê gel û bi vî rengî têkiliya nifşên nû û yên kevin saz dikin. Di 
kilamên şînê de wate û huner di nava hevsengiyekê (denge) de ne. Di çanda kurdî ya devkî de, bi taybetî jî kilamên dengbêjan yên şer û şînan de şopên dîroka kurdan ya nêzik hene. Lewma jî divê em bidin ser şopa kilaman. Her kilamek rê li ber niqaşa dîroka fermî ku li me hatiye ferzkirin vedike. Divê em hîmê çand û dîroka gelê xwe ya nêzik li ser van kilaman deynin. Ew kilaman dikarin hest û ramanên ku pêşiyên me hîs dikirin b ime bidin fêmkirin. Herweha ew kilam dikarin rê li ber pişaftina çand, ziman û muzîka kurdî bigrin. Ji bo vê yekê jî divê kilamên kurdî ji aliyê kurdan ve bêyî ku bêne guhertin werin tomarkirin. Li ser van deng û kilamên tomarkirî divê xebatên akademîk bêne pêşxistin. Divê di zanîngehan de derfeta lêkolînên muzîka kurdî bêne sazkirin. Yan na piştî ku dengbêj bimire ê deng û reng jî tevî çand û dîrokê bi wî re bimirin.

\section{ÇAVKANî}

Bayrak, M. (1993). Kürtler ve Ulusal-Demokratik Mücadeleleri. Ankara: Özge.

Bayrak, M. (2002). Kürt Müziği Mansları ve Şarkıları (Berg 1-3). Ankara: Özge.

Bayrak, M. (2012). Ağıtlama-șarkılarda Dersim Soykırımı. www.politikart1.blogspot.com

Retrieved on June 06, 2015.

Bayrak, M. (2013). Kürtlere Vurulan Kelepçe: Şark Islahat Planı. Ankara: Özge.

Cewerî, F. (2004) Ziman Bingeh û Çavkaniya Edebiyatê Ye. Vesta, Bihar-Havin, 135-138.

Connerton, P. (1999). Toplumlar Nasıl Anımsar? Alaeddin Şenel (Wer.). Stenbol: Ayrıntı.

Çağlayan, H. (2010). Analar, Tanrıçalar, Yoldaşlar. Stenbol: İletişim.

Çelik, A. (2015). Eşîra Xiyan, Bîra Kollektîf û Trawma: Êşeke Patetîk Li Dor Kilama Reben im. Wêje û Rexne, 4, 180-202.

Çifçi, T. (2014). Kilam û Jin. Stenbol: Nûbihar.

Dît, N. (2012). Şiir ve Stran Tahlilleri-4, www.kovarabir.com. Retrieved on January 31, 2012.

Elaldı, Ö. \& Bilgin, D. (2011, 9 Aralık). Ağıtlarda Dersim Soykırımı 2. http://www.ozgurgundem.com/haber/26814/agitlarda-dersim-soykirimi-2

Erbay, V. (2004). Li Ser Wêjeya Kurdî Ya Hemdem Ramanên Kesane. Mahsum Taşkesen (Wer.). Vesta, Bihar-Havîn 2004, 108-112.

Karacan, H. \& Nayman R. (2014). Dengbêj Salihê Qubînî ile Karapetê Xaço'nun Şarkılarında Aşiret Kavgaları: "Fı11̂tê Quto İle Elî Etmankî Kavgası". Dicle Üniversitesi İlahiyat Fakültesi Dergisi, c.16/2, 361 - 388.

Karpat, K. (1971). Türk Edebiyatında Sosyal Konular. İstanbul: Varlık.

Kevirbirî, S. (2009a). Sedsala Qêrinekê Karapetê Xaço. Stenbol: Do.

Kevirbirî, S. (2009b). Fîlitê Quto: Bîst Kilam û Bîst Qewimîn. Stenbol: Do.

Kıran, E. (2011). Derwêşê Evdî. İstanbul: Nûbihar.

Oğuz, C. (2010). Hevpeyvîn: Dengbêj Mihemedê Serhedî. Tîroj, 46, 45-48. 
Özbek, M. (1981). Folklor ve Türkülerimiz İstanbul: Ötüken.

Özcan, M. (2002). Öyküleriyle Dersim Ağıtları 1 (Arekerdox). Ankara: Kalan.

Özcan, M. (2003) Kürdün Gelini (Notalarıyla Tunceli Halk Türküleri ve Oyun Havalarl), Ankara: Kalan.

Özcan, M. (2006). Öyküleriyle Dersim Ă̆ıtları (B. 1-2). Ankara: Kalan.

Porteli, A. (2005). Sözlü Tarihi Farklı Yapan Şey. Kürşat Korkmaz (Wer.). Milli Folklor, 68, 222-234.

Sokolov, Y. M. (2009). Folklor, Tarih ve Kuram. Yetke Özer (Wer.). Ankara: Geleneksel.

Uluçay, Ö. (2006). Dewrêşê Evdî Destanı, Stenbol: Do.

Uzun, M. (2006). Dengbêjlerim. Stenbol: İthaki.

Veroj, S. (1997). Folklora Kurdî. War, 1, 112-132.

Yönetken, H. B. (2003) Tunceli. Kürdün Gelini (Notalarlyla Tunceli Halk Türküleri ve Oyun Havalarl). Mesut Özcan (Edit). Ankara: Kalan. 